\title{
The Analysis of Climate Change in Haiyan County
}

\author{
Wenzheng Yü ${ }^{1}$, Hanxiaoya Zhang ${ }^{2}$, Tianliang $\mathrm{Chen}^{3}$, Jing Liu ${ }^{1}$ and Yanbo Shen ${ }^{4,5 *}$ \\ ${ }^{1}$ School of Applied Meteorology, Nanjing University of Information Science \& Technology, \\ Nanjing, 210000 - China \\ [e-mail: 002030@nuist.edu.cn] \\ 2 Faculty of Science, The University of Auckland, Auckland 1010 - New Zealand \\ [e-mail: hzha734@aucklanduni.ac.nz] \\ ${ }^{3}$ College of Environmental Science and Engineering, Nanjing University of Information Science \& Technology, \\ Jiangsu Nanjing, 210044 - China \\ [e-mail: g1z217@163.com] \\ ${ }^{4}$ Public Meteorological Service Center, China Meteorological Administration, Beijing 100081 - China \\ ${ }^{5}$ Center for Wind and Solar Energy Resources, China Meteorological Administration, Beijing 100081 - China \\ [e-mail: shenyb@cma.gov.cn] \\ *Corresponding author: Yanbo Shen
}

Received June 22, 2020; revised August 6, 2020; accepted September 12, 2020; published October 31, 2020

\begin{abstract}
In this paper, the climate change in Haiyan County in recent decades was analyzed in detail with the methods of moving average, Mann-Kendall non-parametric mutation test and wavelet analysis. According to the variation trend of meteorological factors such as temperature, relative humidity, wind speed, pan evaporation and precipitation in recent decades, the climate of Haiyan County has a tendency of drought, which is becoming more and more serious. From the results of the analysis, the sunshine hours and the air temperature in Haiyan County have an obvious upward trend. The average surface temperature has increased by $2.75{ }^{\circ} \mathrm{C}$ from 1976, and its largest increase occurred in the late 1970s and 1980s. At this stage, the average surface temperature increased by $1.37^{\circ} \mathrm{C}$. The relative humidity has a decreasing trend that has decreased by $2.75 \%$. From 1976 to the present, there are two quasi-3a cycles and one quasi-6a cycle. The precipitation and evaporation showed the opposite change trend, in which the trend of precipitation fluctuated upward, while the trend of evaporation showed a fluctuating downward tendency, which led to the serious loss of water in the feeding area. The wind direction in Haiyan County are mainly from west to east, and its wind speed has a trend of slight increase.
\end{abstract}

Keywords: Haiyan, drought, temperature, precipitation 


\section{General situation of study area}

Haiyan County is located in the northeast of Qinghai Lake Basin at $100^{\circ} 23^{\prime}-101^{\circ} 20^{\prime}$ east longitude, $36^{\circ} 44^{\prime}$ - $37^{\circ} 39^{\prime}$ ' north latitude, with a total area of 4853.08 square kilometers. It belongs to alpine geomorphology, of which the mountainous area is $45 \%$, the beach is $40 \%$, the low hill is $10 \%$, and the Huangshui valley is $5 \%$ [1]. The climate of Haiyan County is plateau sub-arid climate, with arid and windy spring, cool summer, short autumn, and long winter. Without an absolute frost period, Haiyan County has an annual average temperature of about $1.5^{\circ} \mathrm{C}$, an annual sunshine time of about 2980 hours, an annual precipitation of about $400 \mathrm{~mm}$, and an annual evaporation of about $1581.75 \mathrm{~mm}$ [2]. Hail, frost, drought, wind and sand disasters in this place are frequent. The study area of this paper is the desertified land at the border of Haiyan County and Qinghai Lake [3].

\section{Research methods and data sources}

The methods of moving average, Mann-Kendall non-parametric mutation test and wavelet analysis were used to analyze the evolution law and the trend of climate factors in the 40 years, including average annual temperature, average annual relative humidity, average annual wind speed, average annual precipitation and average annual surface temperature. For each correlation analysis, a P value was calculated and provided to indicate the whether the trend observed for the climatic trend was significant. In this study, $\mathrm{P}<0.05$ ( $95 \%$ confidence level) is considered significant [4]. The data in this study were retrieved from China Meteorological Data Network [5].

\subsection{Moving average analysis}

The moving average analysis is one of the basic curve fitting methods, equivalent to a low-pass filter [6] [7]. The smoothed curves of the time series can be used to observe the variation trends. For a series ' $x$ ' with the sample size ' $n$ ', the moving average formula can be expressed as:

$$
\widehat{X_{J}}=\frac{1}{k} \sum_{i=1}^{k} X_{i+j-1}(\mathrm{j}=1,2, \cdots \cdot \mathrm{n}-\mathrm{k}+1)
$$

In the formula, $\mathrm{k}$ is the moving length, which is decided based on the specific problem and the sample size. $n-k+1$ smoothed values can be obtained with $n$ samples. After the moving average analysis, the periods shorter than the moving length are greatly weakened [8]. As a result, the trends are better indicated through the smoothed curves from the time series.

\subsection{Mann-Kendall non-parametric mutation test}

The advantage of Mann-Kendall non-parametric mutation test is that it can be applied to samples that do not follow a pattern of distribution, while negating the impacts of irregular data [9] [10]. For a time series ' $x$ ' with a sample size ' $n$ ', the formula can be expressed as:

$$
S_{k}=\sum_{i=1}^{k} r_{i}(\mathrm{k}=2,3, \cdots \cdot \mathrm{n})
$$


Where:

$$
r_{i}=\left\{\begin{array}{r}
+1 \\
0
\end{array} \text { when } x_{i}>x_{j} \quad(\mathrm{j}=1,2, \cdots, \mathrm{i})\right.
$$

The $S_{k}$ sequence is the cumulative number of data where the value at time $i$ is greater than the value at time $\mathrm{j}$. Assuming the time series is independent and random, the formula for magnitude can be expressed as:

$$
U F_{k}=\frac{\left[s_{k}-E\left(s_{k}\right)\right]}{\sqrt{\operatorname{Var}\left(s_{k}\right)}}(\mathrm{k}=1,2, \cdots, \mathrm{n})
$$

In the formula, $\mathrm{UF}_{1}=0 ; \mathrm{E}\left(\mathrm{S}_{\mathrm{k}}\right)$ and $\operatorname{Var}\left(\mathrm{S}_{\mathrm{k}}\right)$ are the mean and variance of $\mathrm{S}_{\mathrm{k}}$, which could be calculated with the following formula when the data are independent from each other and distributed continually:

$$
\begin{aligned}
\mathrm{E}\left(s_{k}\right) & =\frac{n(n+1)}{4} \\
\operatorname{Var}\left(s_{k}\right) & =\frac{n(n+1)(2 n+5)}{72}
\end{aligned}
$$

UF can be compared with the critical value $U_{a}$, which can be obtained by querying the normal distribution table $\left(\mathrm{U}_{0.05}=1.96\right)$ and indicate the significance level of the trend or mutation point observed in the test [11]. If $|\mathrm{UF}|>\mathrm{U}_{0.05}$, then the trend is significant with $95 \%$ confidence level. The approximate abrupt change point can then be located close to the intersection of UF and UB curves, and the abrupt change point is significant if the intersection is within the confidence interval [12].

\subsection{Wavelet analysis}

The time series in this study were studied with the wavelet analysis expressed as [13] :

$$
\psi_{0}(t)=\pi^{-1 / 4} e^{i \omega_{0} t} e^{-t^{2} / 2}
$$

In the equation, $t$ is the time; $\omega_{0}$ is the dimensionless frequency. When $\omega_{0}=6$, the scale term and period term are interchangeable. The wavelet analysis incorporated Morlet wavelet transform, which had the benefit of non-orthogonality as well as a good balance between the localization of time and frequency [14] [15]. The continuous wavelet of the discrete time series $\mathrm{x} \_\mathrm{n}(\mathrm{n}=1,2, \cdots, \mathrm{N})$ with equal time step $\delta \_\mathrm{t}$ is re-defined as the convolution of the scale change of the wavelet function $\psi \_0$ and the converted $\mathrm{x} \_\mathrm{n}$ :

$$
W_{n}^{X}(s)=\sqrt{\frac{\delta_{t}}{s}} \sum_{n=0}^{N-1} x_{n} \psi^{*}\left[\frac{\left(n^{\prime}-n\right) \delta_{t}}{s}\right]
$$


Where * represents the conjugate complex number; $\mathrm{n}$ is the total number of data in the time series; $\left(\delta_{-} t \mathrm{~s}\right)^{\wedge}(1 / 2)$ is a factor used for standardization of the wavelet function, so that the wavelet function has on each wavelet scale s Unit energy. By converting the wavelet scale s and localizing along the time index $\mathrm{n}$, a graph showing the fluctuation characteristics of the time series on a certain scale and its changes over time can be obtained. The global wavelet spectrum can then be obtained with the following formula:

$$
\bar{W}^{2}(s)=\frac{1}{N} \sum_{n=0}^{N-1}\left|W_{n}(s)\right|^{2}
$$

\section{Analysis of climate change characteristics}

\subsection{Characteristics of temperature variation}

As shown in Fig. 1 (I), the average air temperature in Haiyan County has shown an obvious upward trend in the past 40 years, in which it increased by $0.03^{\circ} \mathrm{C} / \mathrm{a}$ in the $1980 \mathrm{~s}, 0.01{ }^{\circ} \mathrm{C} / \mathrm{a}$ in the 1990s, and $0.04{ }^{\circ} \mathrm{C} / \mathrm{a}$ since the beginning of the 21 st century. The lowest temperature increase in Haiyan County was $-0.43{ }^{\circ} \mathrm{C}$ in 1976 , and the highest was $1.92{ }^{\circ} \mathrm{C}$ in 1998 . The average temperature increasing rate was $0.07{ }^{\circ} \mathrm{C} / \mathrm{a}$. The correlation analysis showed the increasing trend for the mean temperature was significant $(\mathrm{P}<0.01)$. As shown in Fig. 1 (II), the seasonal distribution of mean air temperature was obvious, and the peak value appears in July or August, indicating the characteristics of a normal distribution.

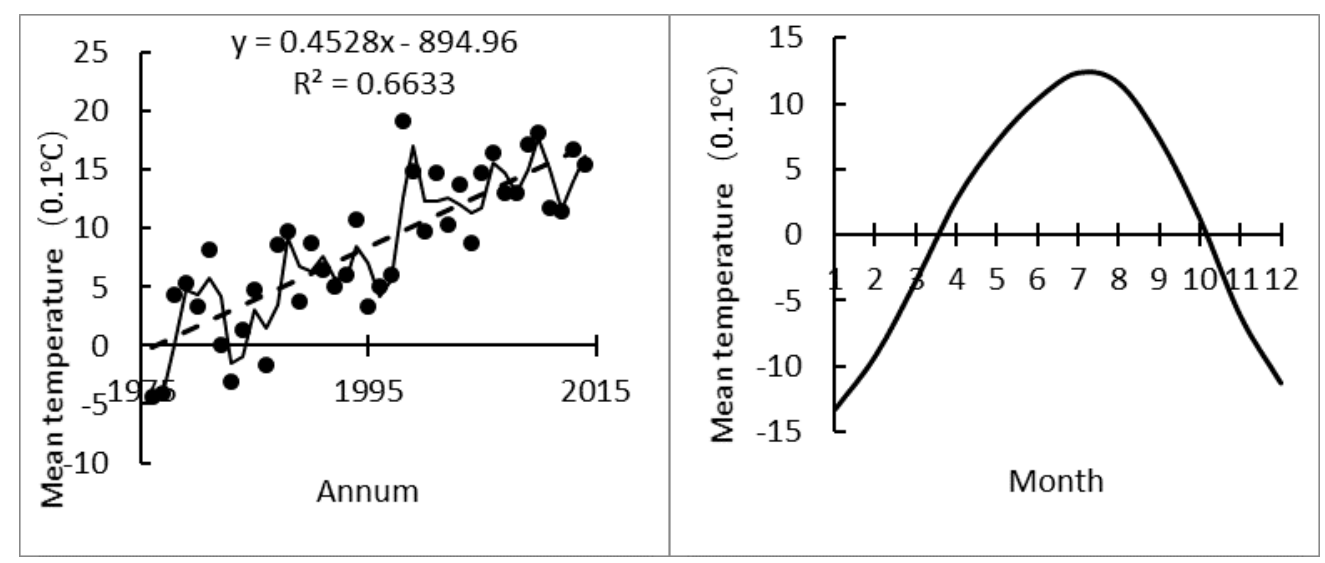

(I) Inter-annual variation

(II) Intra-annual variation

Note: (I) the dotted line represents the linear trend, and the solid line represents a two-year moving average.

Fig. 1. Variation characteristics of mean temperature

As shown in Fig. 2, the mean relative humidity in Haiyan County fluctuated inter-annually in the past 40 years, but an overall decreasing trend was observed. At the end of the 1970s, there was a rapid downward trend, with an annual decrease of $2.0 \%$. From the 1980s to the beginning of the 21st century, the mean relative humidity showed a slight increase of $6.2 \%$, but decreased rapidly after 2003. And the average relative humidity of the 2010s had dropped 
to a similar level to the lowest level in the late 1970s and early 1980s. Overall, the average annual decrease of the mean relative humidity was $0.71 \%$, with the maximum value being $66.2 \%$ in 1989 , and the minimum being $54.8 \%$ in 2013 . The correlation analysis showed the overall trend for the mean relative humidity in Haiyan County was not significant $(\mathrm{P}>0.1)$.

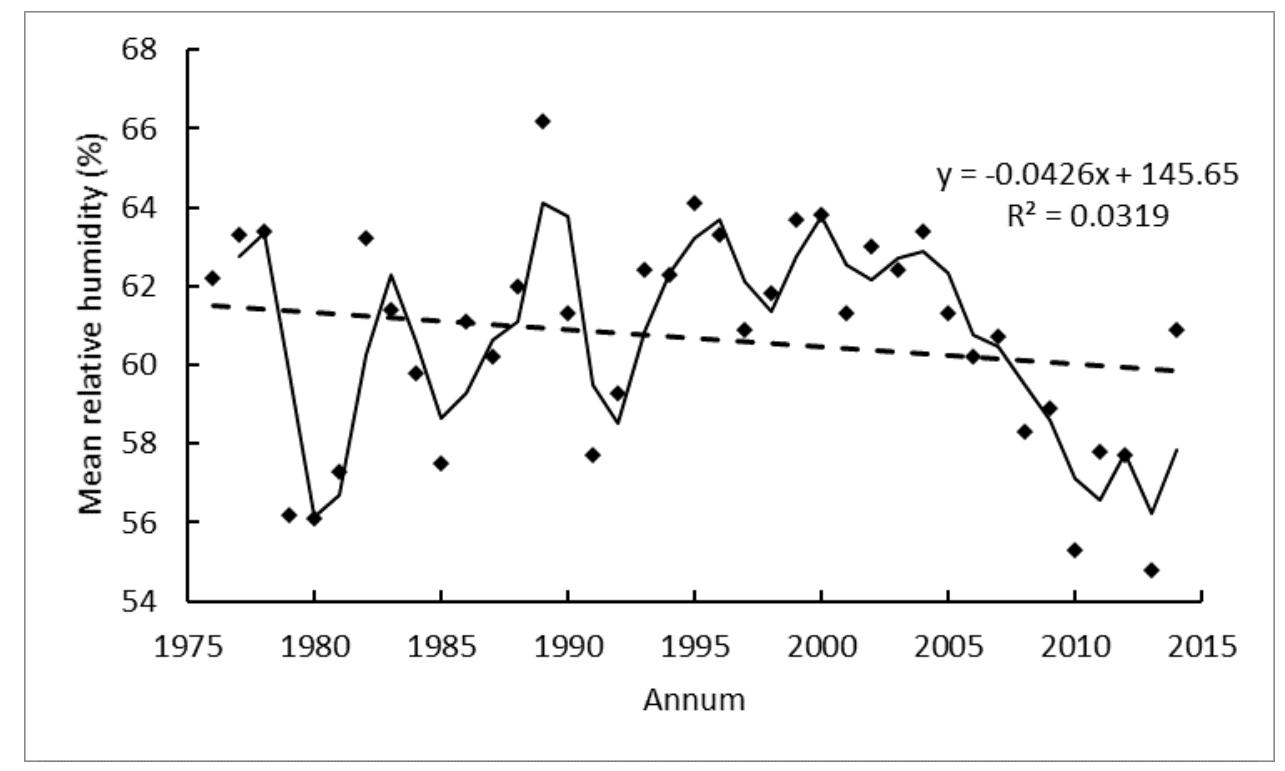

Note: the dotted line in the diagram represents the linear trend, and the solid line represents the two-year moving average

Fig. 2. Inter-annual variation characteristics of mean relative humidity

\subsection{Variation characteristics of mean wind speed}

As shown in Fig. 3 (I), from the perspective of year-to-year variation, the annual average wind speed in Haiyan County has shown a slight increase over the past 40 years. In the late 1970s, the annual average wind speed increased, with an annual increase of $0.17 \mathrm{~m} / \mathrm{s}$. From the $1980 \mathrm{~s}$ to the early 1990s, the annual average wind speed decreased by the value of $0.04 \mathrm{~m} / \mathrm{s}$ per year. From the beginning of the 1990s to the middle of the 1990s, there was no drastic change to the annual average wind speed. From the mid-1990s to 2014, the annual average wind speed increased by at the rate of $0.17 \mathrm{~m} / \mathrm{s}$ per year, doubled in 2014. During the studied period, the highest annual average wind speed was $5.8 \mathrm{~m} / \mathrm{s}$ in 2014; the lowest value was $2.5 \mathrm{~m} / \mathrm{s}$ that appeared in the early to mid-1990s; the range of variation was $2.3 \mathrm{~m} / \mathrm{s}$. The correlation analysis results showed the variation trend for the mean wind speed was not significant $(\mathrm{P}>0.1)$. Fig. 3 (II) showed the monthly variation of average wind speed in Haiyan County based on the compiled yearly data of the studied period. From the figure, it was found that the average wind speed in Haiyan County remained above $2.5 \mathrm{~m} / \mathrm{s}$ throughout the year. Over $3 \mathrm{~m} / \mathrm{s}$ was recorded from February to May, reaching the peak in March and April. 


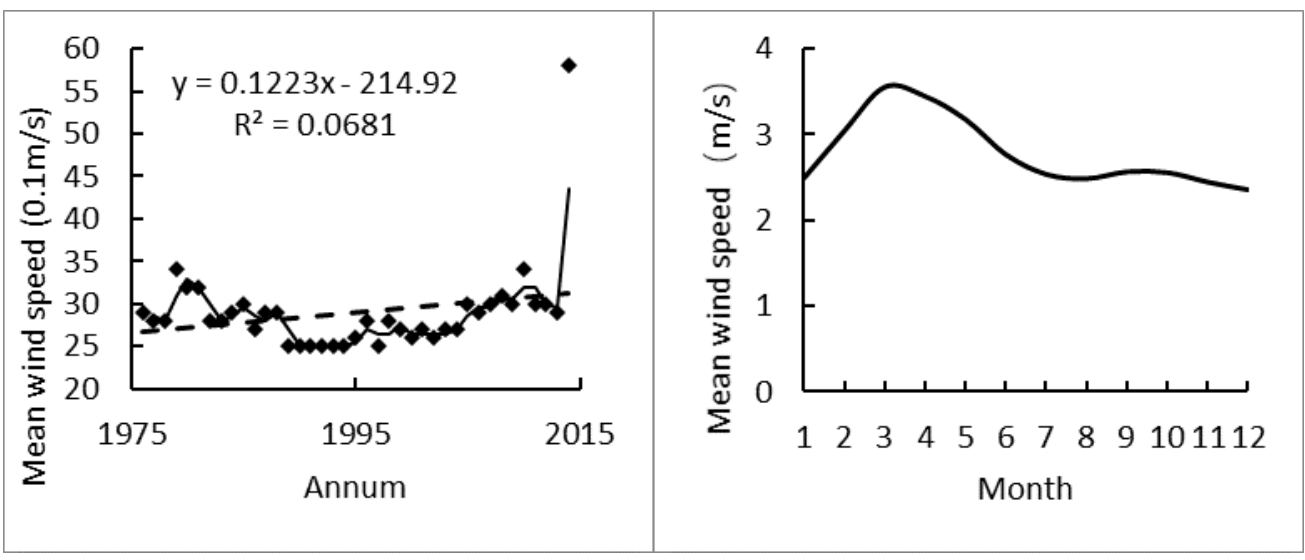

(I) Inter-annual variation

(II) Intra-annual variation

Note: (I) The dotted line represents the linear trend and the solid line represents a two-year moving average.

Fig. 3. Variation characteristics of mean wind speed

\subsection{Variation characteristics of pan evaporation}

As shown in Fig. 4, the pan evaporation in Haiyan County showed a fluctuating downward trend from 1975 to 2010, with a decrease rate of $1.2 \mathrm{~mm} / \mathrm{a}(\mathrm{P}>0.1)$. The pan evaporation increased rapidly in the middle and late 1970s, with an average annual growth rate of 77.4 $\mathrm{mm} / \mathrm{a}$. However, from the beginning of 1980s to the middle and late 1990s, it decreased at the rate of $17.6 \mathrm{~mm}$ per year. From the late 1990s to 2010, it showed an upward trend, and its average increase rate was $9.0 \mathrm{~m}$. During the study period, the range of pan evaporation in Haiyan County has reached $391.2 \mathrm{~mm}$, with a maximum value of $1673.5 \mathrm{~mm}$ in 1979 and a minimum value of $1282.3 \mathrm{~mm}$ in 1989 . The correlation analysis showed that the overall decreasing trend of pan evaporation was not significant $(\mathrm{P}>0.1)$.

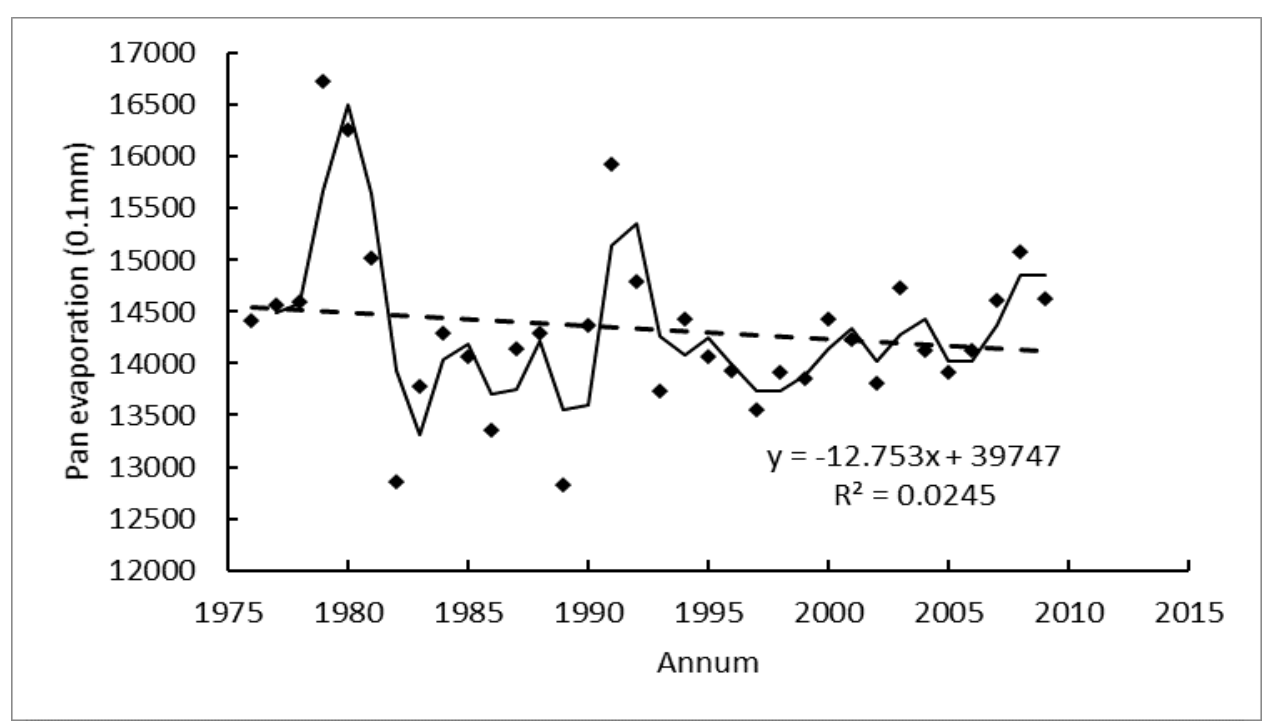

Note: the dashed line in the diagram represents a linear trend, and the solid line represents a two-year moving average

Fig. 4. Interannual variation characteristics of small evaporation 


\subsection{Variation characteristics of sunshine duration}

During the study period, a small increasing trend as shown for the annual sunshine hours in Haiyan County (Fig. 5), the increasing rate being $1.3 \mathrm{~h} / \mathrm{a}$. In the middle and late period of 1970s, the annual sunshine hours increase rapidly, with an average increase rate of $98.8 \mathrm{~h} / \mathrm{a}$. However, from the 1980s to the early 1990s, the annual sunshine hours decreased at the rate of $34.7 \mathrm{~h} / \mathrm{a}$. In the 1990s, another increasing trend was observed at the rate of $30.4 \mathrm{~h} / \mathrm{a}$. In the 21st century, the annual sunshine hours in Haiyan County decreased $12.9 \mathrm{~h}$ on average per year. During the study period, the maximum annual sunshine hours was recorded in 1997 (3118.3h), and the minimum was recorded in 1976 (2627.9h), the range of variation being $490.4 \mathrm{~h}$. The correlation analysis showed that the overall increasing trend of the sunshine duration was not significant $(\mathrm{P}>0.1)$.

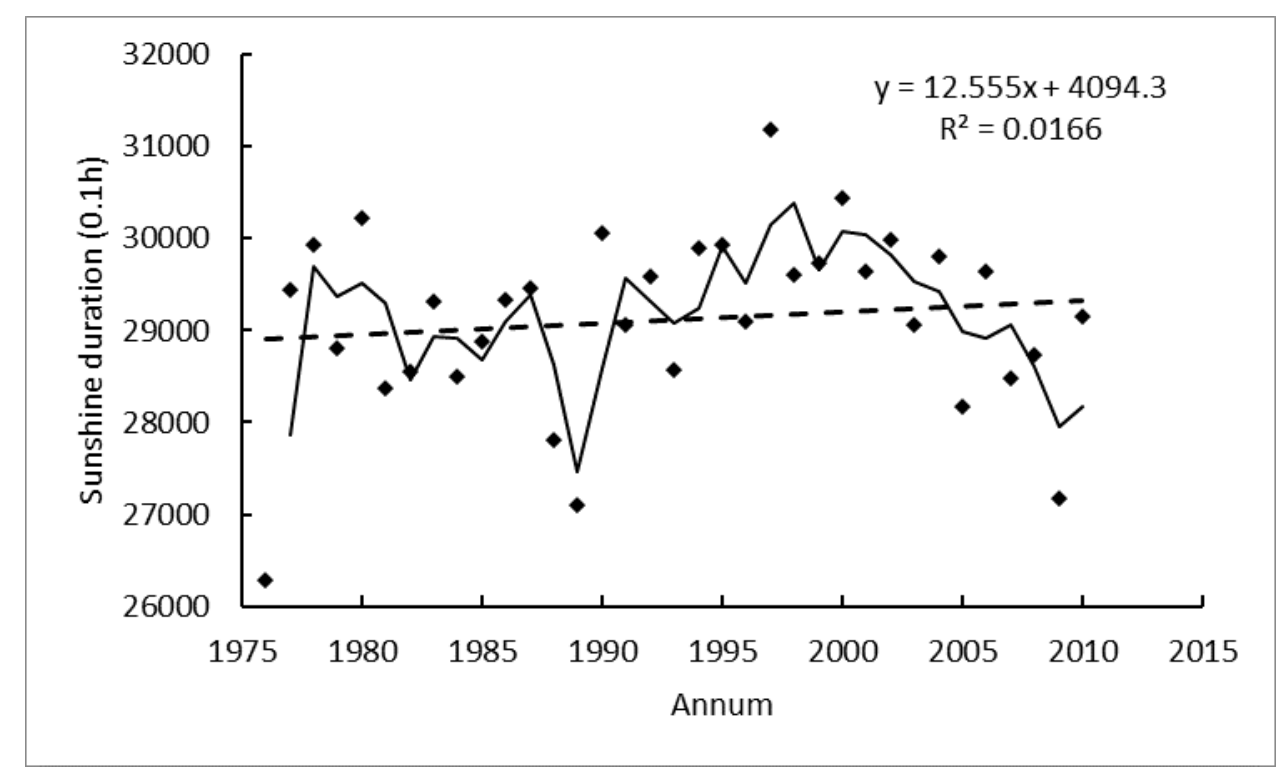

Note: the dashed line in the diagram represents the linear trend, and the solid line represents the two-year moving average

Fig. 5. Inter-annual variation characteristics of sunshine hours

\subsection{Characteristics of precipitation variation}

As shown in Fig. 6 (I), there was a slight increasing trend for the annual precipitation in Haiyan county at the rate of $0.64 \mathrm{~mm} / \mathrm{a}$. The maximum value was $522.3 \mathrm{~mm}$ in 1989; the minimum value was $248.2 \mathrm{~mm}$ in 2000; and the range of variation was $274.1 \mathrm{~mm}$. In the mid and late 1970s, the annual precipitation in Haiyan County decreased, with an average decrease rate of $19.3 \mathrm{~mm} / \mathrm{a}$. However, in the 1980s, it increased at the rate of $27.6 \mathrm{~mm} / \mathrm{a}$. Since the 1990s, the annual precipitation has decreased, with an average decrease rate of $10.5 \mathrm{~mm} / \mathrm{a}$. In the 21st century, the annual precipitation in Haiyan County increased at $27.9 \mathrm{~mm} / \mathrm{a}$, but a downward trend occurred since 2009 with a decrease rate of $30.1 \mathrm{~mm} / \mathrm{a}$. The correlation analysis indicated that the overall increasing trend of precipitation in Haiyan County was not significant ( $\mathrm{P}>0.1$ ). Fig. 6 (II) demonstrated the distribution of precipitation on a monthly basis. Overall, the graph followed the normal distribution. A seasonal distribution was also 
observed, with the majority of the precipitation taking place in summer and autumn, peaking during July and August.

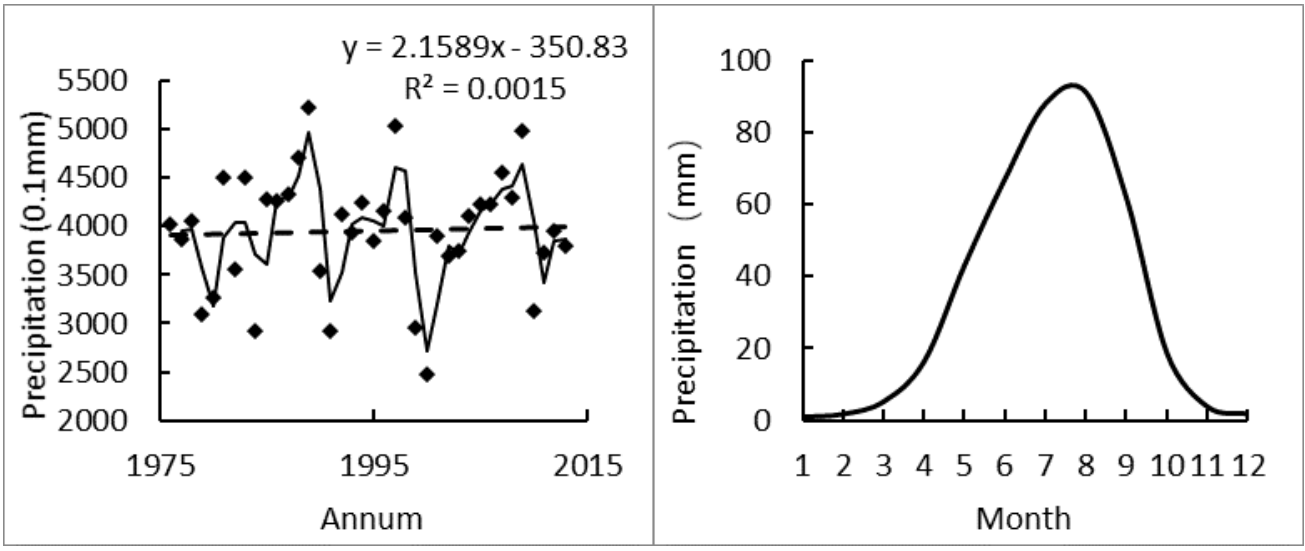

(I) Inter-annual variation

(II) Intra-annual variation

Note: (I) The dotted line represents a linear trend, and the solid line represents a two-year moving average.

Fig. 6. Variation characteristics of precipitation

\subsection{Variation characteristics of mean surface temperature}

As shown in Fig. 7, an overall increasing trend was observed for the mean surface during the study period at the rate of $0.07^{\circ} \mathrm{C} / \mathrm{a}(\mathrm{P}<0.01)$. The average surface temperature of Haiyan County was the lowest at $2.98{ }^{\circ} \mathrm{C}$ in 1976 and the highest at $6.03^{\circ} \mathrm{C}$ in 2013 . From 1976 to 2013, the average surface temperature of Haiyan County increased by $3.05^{\circ} \mathrm{C}$.

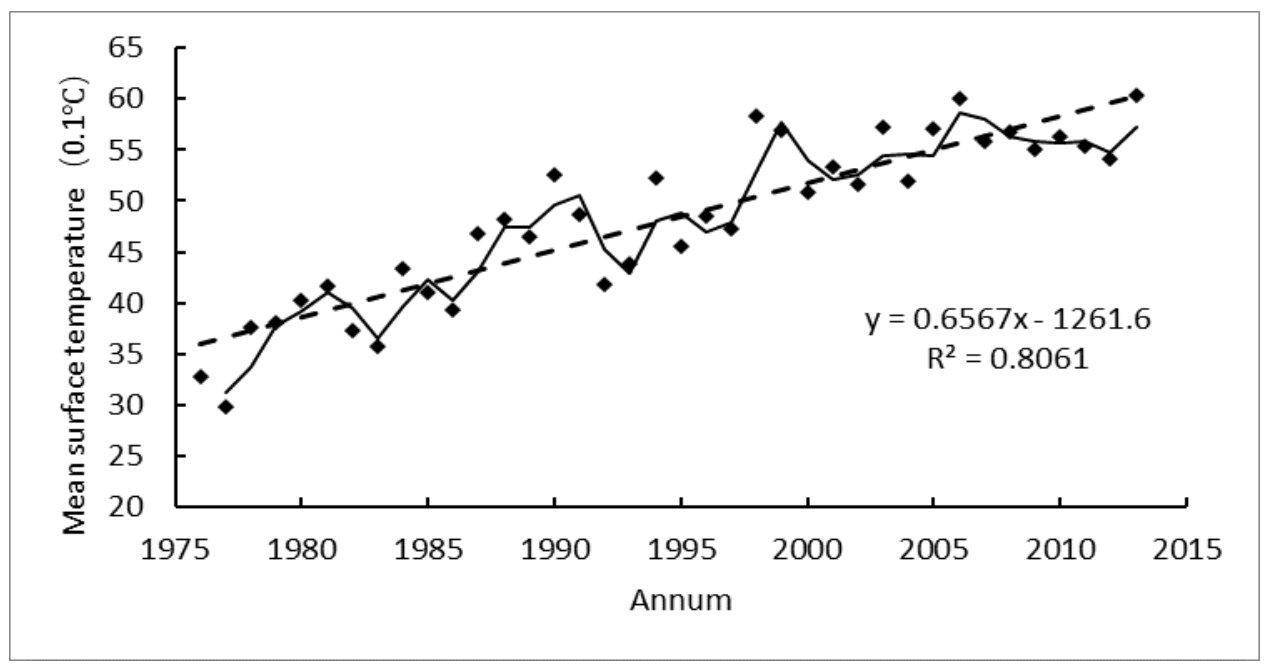

Note: The dashed line in the diagram represents the linear trend, and the solid line represents the two-year moving average

Fig. 7. Interannual variation characteristics of mean surface temperature 


\section{Characteristic analysis of climatic cycle}

Fig. 8 showed the power spectrum of meteorological factors in Haiyan County in the recent decades transformed by Morlet wavelet. The larger the wavelet power in the graph, the denser the contour lines were. The areas surrounded by dotted lines were the period that passed the Gaussian red noise test $(\mathrm{P}<0.05)$; the areas surrounded by the inverted cone lines (in the shape of *) were the period where the edge effect was removed. The wavelet power spectrum of the rest of the areas were influenced by the edge effect, hence there was uncertainty in the periodic characteristics in these areas.

As shown in Fig. 8(a), there were two significant quasi-3a periods and one significant quasi-4a period in the mean temperature time series in Haiyan County, and the intensity of these periods varied inter-annually. The two quasi-3a periods appeared in 1976-1990 and 1998-2001 respectively, with the former stronger than the latter. During the first quasi-3a period, the mean annual temperature experienced a process of "rise-drop-rise", with the temperature rising by $1.3^{\circ} \mathrm{C}$. During the second quasi-3a spectrum period, the mean annual temperature decreased $0.44{ }^{\circ} \mathrm{C}$. The quasi-4a cycle occurred during the period of 1996-1999, where the mean annual temperature increased $0.98{ }^{\circ} \mathrm{C}$. The wavelet power spectrum analysis also detected the presence of quasi-12a and quasi-24a cycles, but none was significant $(\mathrm{P}>0.1)$

As shown in Fig. 8(b), there were significant quasi-2 4 a and quasi-8a periods in the precipitation time series in Haiyan County, and the intensity of these periods changed along with the annum. The periodic characteristics of the quasi-2 4a periods appeared during 1978-2003, where the annual precipitation fluctuated four times and overall decreased $32 \mathrm{~mm}$. The periodic characteristics of the quasi-3a spectrum appeared in the period of 2007-2012, where its annual precipitation experienced the process of "increase-decrease-increase", and overall decreased $59.9 \mathrm{~mm}$. The periodic characteristics of the quasi-8a spectrum appeared in the period of 1983 -2001, where the annual precipitation decreased $61.4 \mathrm{~mm}$. The quasi-2 4a spectrum was stronger in $1990 \sim 2001$ and weaker in other years.

As shown in Fig. 8(c), there were two significant quasi-3a periods and one significant quasi-6a period in the mean relative humidity time series in Haiyan County, and the intensity of the periods changed along with the annum. The periodic characteristics of the quasi-3a periods appeared during 1984-1993 and 2009-2014. During the first quasi-3a spectrum period, the mean annual relative humidity experienced the process of "decrease-increase-decrease", and overall increased $0.58 \%$. During the second quasi-3a period, the mean annual relative humidity fluctuated twice, and the total amount decreased by $6.21 \%$. The characteristic of quasi-6a the period period appeared during 1976-1996, and its mean annual relative humidity experienced four fluctuations, and overall increased $1.1 \%$. Its spectrum was stronger from the end of 1970s to the early 1980s, but weaker in other years.

As shown in Fig. 8(d), there were significant quasi-3a and quasi-3 4a periods in the time series of sunshine hours in Haiyan County, and the intensity of these periods changed along with the annum. The quasi-3a period appeared in 1976-1984, the annual sunshine hours experienced three fluctuations, and its total amount increased 221.5 hours. The quasi-3 4a periods, appearing during 1984-2010, was stronger in the early 1990s but weaker in other years, and the annual sunshine hours increased by 66.2 hours. At the same time, it was detected by wavelet power spectrum analysis in the time series of sunshine hours in Haiyan County that periods between 16a and 32a were present, but not significant $(\mathrm{P}>0.1)$. 
Fig. 8(e) showed one significant quasi-4a period in the time series of mean annual wind speed in Haiyan County. Its periodic characteristics appeared in the period of $2008 \sim 2014$, and the mean annual wind speed overall increased $2.7 \mathrm{~m} / \mathrm{s}$. The wavelet power spectrum analysis also quasi-12a and quasi-24a periods in the series, but not significant $(\mathrm{P}>0.1)$.

Fig. 8(f) showed one quasi-4 6a period in the annual pan evaporation time series in Haiyan County. The periodic characteristics appeared during 1976-1996, and the annual pan evaporation decreased $48.9 \mathrm{~mm}$. Meanwhile, the wavelet power spectrum analysis also detected the presence of quasi-6a and quasi-8a scale periods in the series, but not significant $(\mathrm{P}>0.1)$.

Finally, no characteristic period was observed in the surface temperature series in Haiyan County.

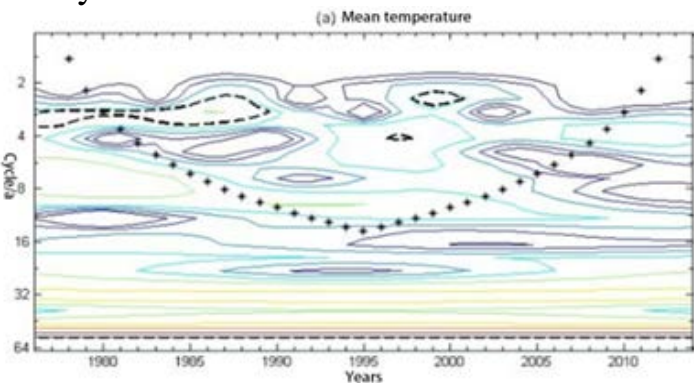

(a) Mean temperature

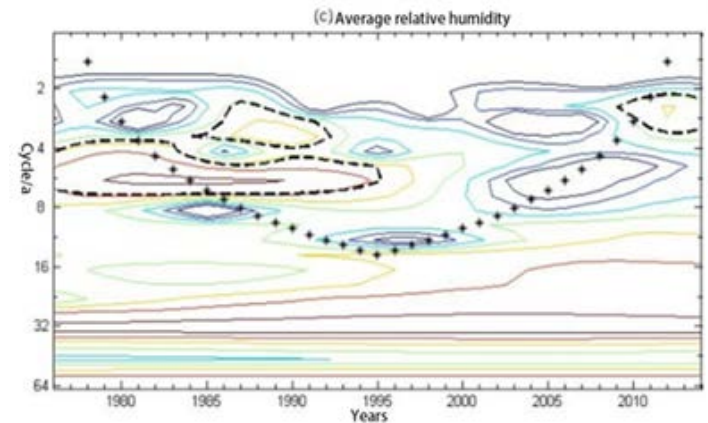

(c) Mean relative humidity

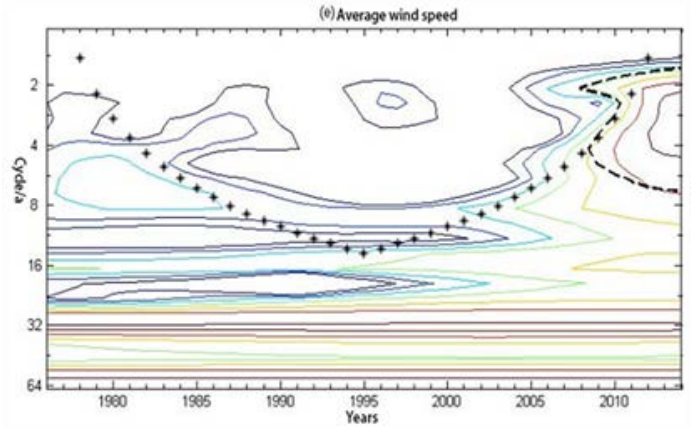

(e) Mean wind speed

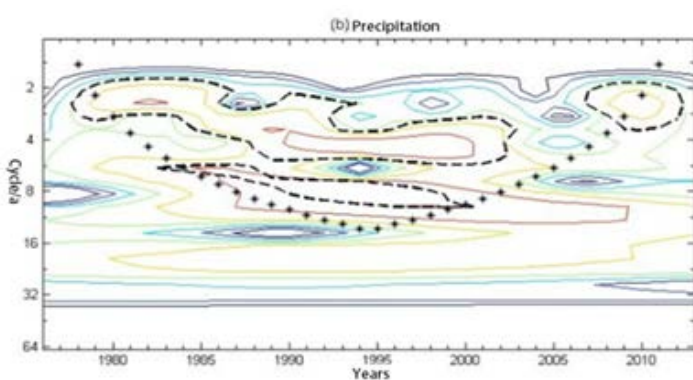

(b) Precipitation

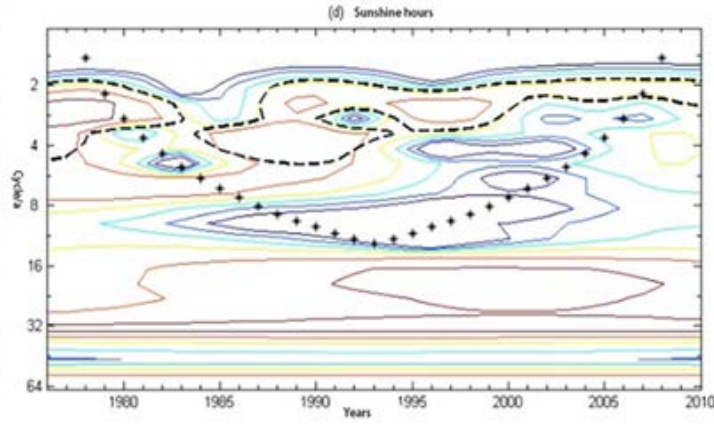

(d) Sunshine hours

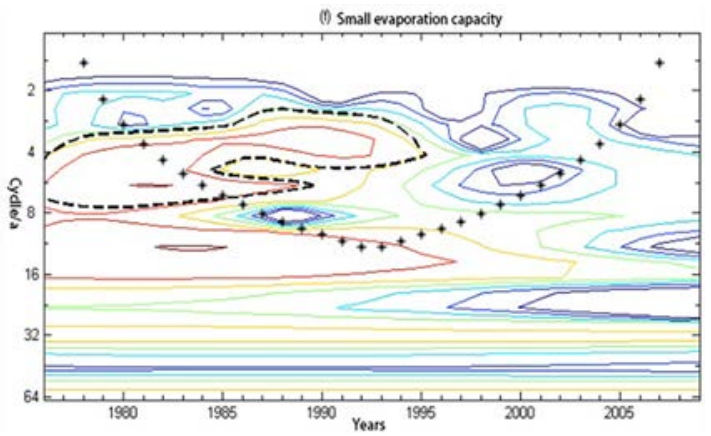

(f) Pan evaporation

Fig. 8. Wavelet power spectrum of meteorological factors in Haiyan County 


\section{Mann-Kendall non-parametric mutation test for climate change}

As shown in Fig. 9(I), the mean annual temperature in Haiyan County showed a clear upward trend, which greatly exceeded the critical line of significant level 0.05 after 1990, indicating that the trend was significant. This was consistent with the previous analysis of the inter-annual variation of mean temperature. According to the intersection point of UF curve and UB curve, the year 1993 might be the abrupt point that the average temperature of Haiyan County becomes rising, but the mutation point was not within the confidence interval, so its credibility needed to be further discussed.

As shown in Fig. 9(II), the average surface temperature of Haiyan County showed a "downward-upward" trend in the time series. Since 1983, the rising trend of surface temperature gradually increased, and exceeded the critical line of significant level 0.05 after 1986. Therefore, the rising trend of the surface temperature was significant, which was consistent with the analysis of the inter-annual variation of the surface temperature before. According to the intersection point of UF curve and UB curve, the year 1989 might be the abrupt point that the average surface air temperature of Haiyan County becomes rising, but the mutation point was not within the confidence interval, so its reliability needed to be further discussed.

From Fig. 9 (III) (IV) (V) (VI) (VII), it can be seen that the trends of precipitation, mean relative humidity, mean wind speed, pan evaporation and sunshine hours in Haiyan County were all in a state of constant fluctuation. By and large these trends did not exceed the critical line of significant level 0.05 , indicating that the variation trends of these meteorological factors were not significant.

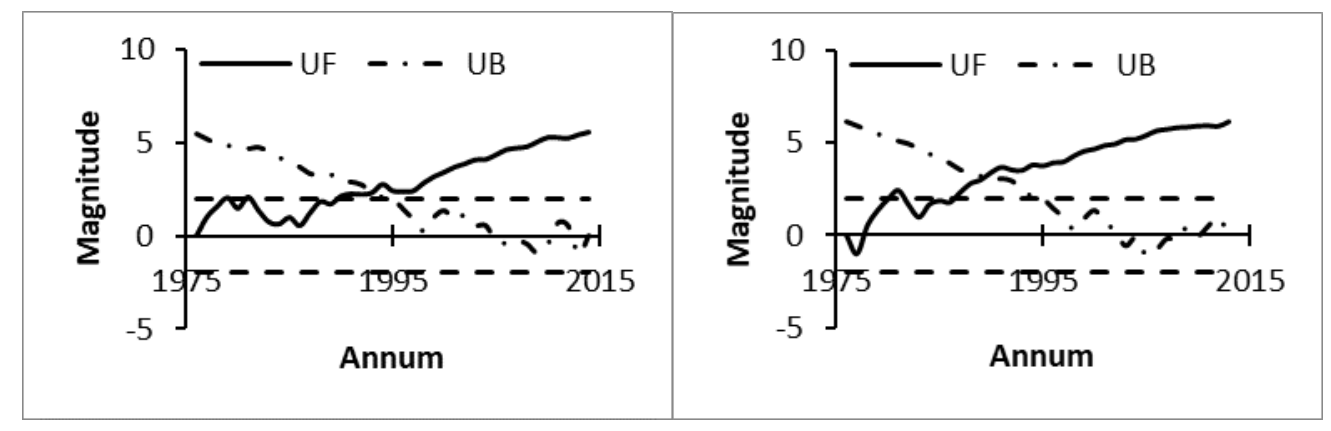

(I) Mean annual temperature

(II ) Mean surface temperature

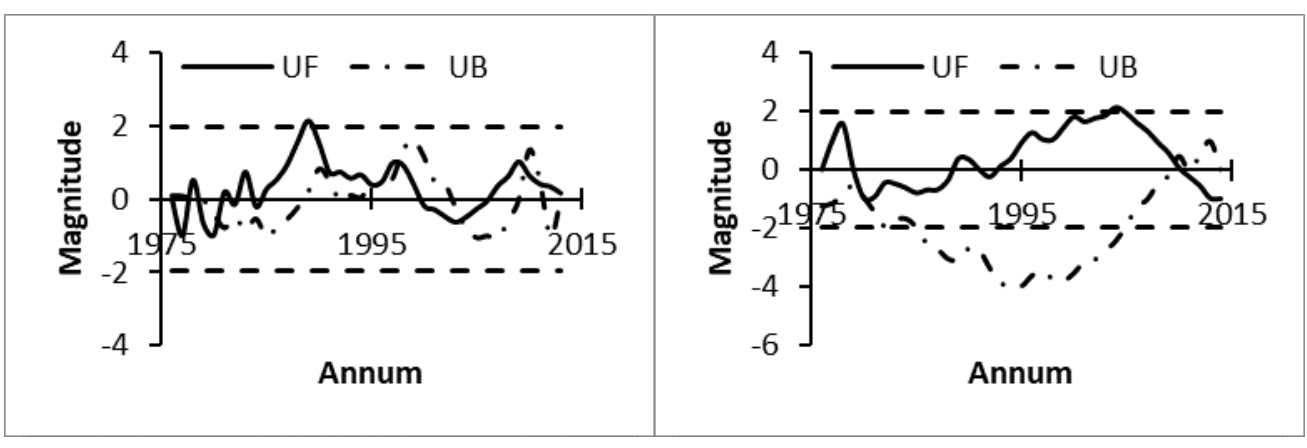

(III) Precipitation

(IV) Mean relative humidity 


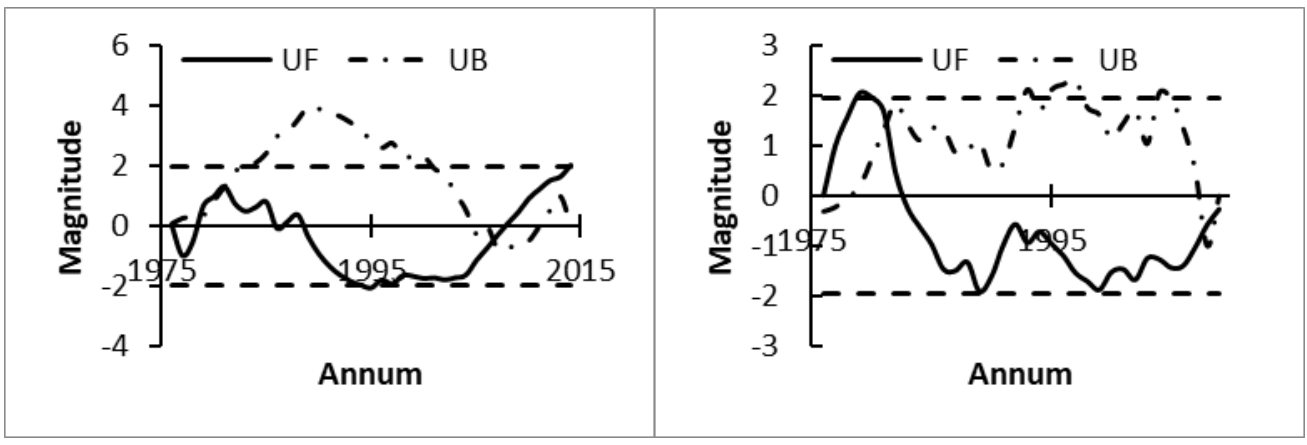

(V) Mean annual wind speed

(VI) Pan evaporation

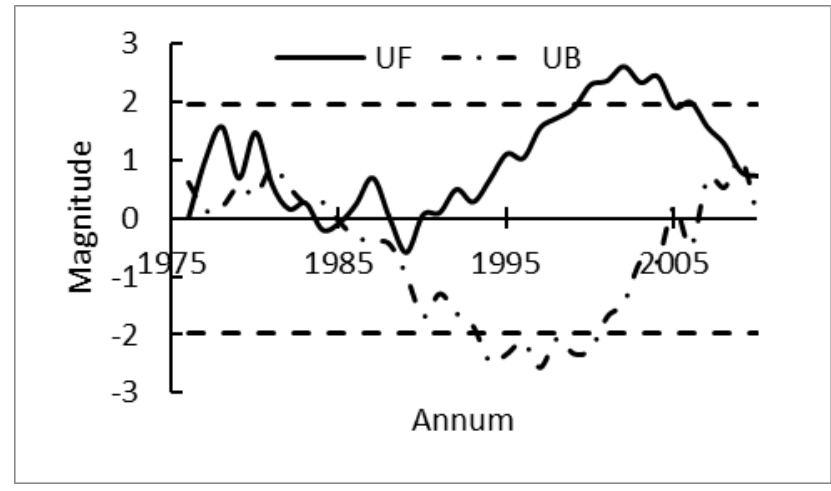

(VII) Sunshine hours

Note: the dotted line in the diagram represents the critical line for $95 \%$ confidence

Fig. 9. Mann-Kendall statistics curve of meteorological factors in Haiyan County

\section{Conclusion}

In this paper, the climate change in the study area in the recent decades was analyzed in detail by means of moving average, Mann-Kendall non-parametric mutation test and wavelet analysis. According to the results of climate change analysis, the sunshine hours and air temperature in Haiyan County showed an obvious upward trend, and the average temperature showed a significant quasi-3a scale cycle and increased by $1.31^{\circ} \mathrm{C}$ in the late $1970 \mathrm{~s}$ and $1980 \mathrm{~s}$. After 1990's, the rising trend of average temperature slowed down, and since 2000, the average temperature has risen $0.06{ }^{\circ} \mathrm{C}$. The average surface temperature has increased by $2.75^{\circ} \mathrm{C}$ since 1976 , and the maximum was $1.37^{\circ} \mathrm{C}$, which was occurred in the late $1970 \mathrm{~s}$ and 1980s. The relative humidity in Haiyan County has a downward trend. Since 1976 the relative humidity has decreased by $2.75 \%$, and at this stage it also occurred two quasi-3a cycles and one quasi-6a cycle. In addition, precipitation and evaporation in Haiyan County showed a reverse trend, in which precipitation fluctuated upward, while evaporation fluctuated downward. But in recent decades, the average annual precipitation in Haiyan County was $395.5 \mathrm{~mm}$, and the average annual evaporation was $1433.7 \mathrm{~mm}$. As for the smaller precipitation, the average difference between precipitation and evaporation reached $1035 \mathrm{~mm} / \mathrm{a}$, which led to the serious water loss. Additionally, the wind direction of Haiyan County in recent decades was mainly from west to east, and it has a trend of slight increase. 
Therefore, the climate of Haiyan County has a trend of drought, which is becoming more and more serious, according to the change trend of meteorological elements such as temperature, relative humidity, wind speed, evaporation and precipitation of Haiyan County in recent decades.

\section{Acknowledgement}

The authors would like to appreciate the support for this study by the Second Tibetan Plateau Scientific Expedition and Research Program (STEP) (Grant No. 2019QZKK0804).

\section{References}

[1] C. Y. Huo, "Measures of Natural Forest Resource Utilization and Guarantee of Haiyan County," Modern Agricultural Technologies, issue 10, pp.177-179, 2014. Article (Web link).

[2] H. T. Liu, "Research on the Correlation between Desertification and Climate Change in Qinghai Lake Basin,” Nanjing University of Information Science \& Technology, 2017. Article (Web link).

[3] W. H. Ma, "Effect and Problems of Desertification Control in Alpine Sand Area of Haiyan County,” Qinghai Agriculture and Forestry Technologies, issue 4, pp.66-68, 2019. Article (Web link).

[4] P. Sedgwick, “Confidence intervals, P values, and statistical significance,” Bmj, pp.350, February 27, 2015. Article (CrossRef Link).

[5] China Meteorological Administration, "Meteorological data of Haiyan County," China Meteorological Data Network, 2020. Database (Web link).

[6] S. W. Smith, “Moving Average Filters,” Digital Signal Processing, pp.277-284, 2003. Article (CrossRef Link).

[7] M. Islam, H. S. Che, M. Hasanuzzaman and N. Rahim, "Energy demand forecasting," Energy for Sustainable Development, pp.105-123, 2020. Article (CrossRef Link).

[8] R. J. Hyndman and G. Athanasopoulos, "6.2 Moving Averages," Forecasting: Principles and practice, OTexts, Heathmont, Victoria, 2018. Article (Web link).

[9] R. Bouza-Deaño, M. Ternero-Rodríguez and A. Fernández-Espinosa, "Trend study and assessment of surface water quality in the Ebro River (Spain),” Journal of Hydrology, 361(3-4), pp.227-239, 2008. Article (CrossRef Link).

[10] R. Li, H. Huang, G. Yu, H. Yu, A. Bridhikitti and T. Su, "Trends of Runoff Variation and Effects of Main Causal Factors in Mun River, Thailand During 1980-2018,” Water, 12(3), pp.831, 2020. Article (CrossRef Link).

[11] Z. Yanming, W. Jun and W. Xinhua, "Study on the Change Trend of Precipitation and Temperature in Kunming City Based on Mann-Kendall Analysis,” Advances in Intelligent and Soft Computing Future Computer, Communication, Control and Automation, pp.505-513, 2011. Article (CrossRef Link).

[12] X. Tang, J. Li, X. Lv and H. Long, "Analysis of the Characteristics of Runoff in Manasi River Basin in the Past 50 Years," Procedia Environmental Sciences, 13, pp.1354-1362, 2012. Article (CrossRef Link).

[13] G. Hariharan, "Wavelet Analysis-An Overview," in Proc. of Wavelet Solutions for Reaction-Diffusion Problems in Science and Engineering, Forum for Interdisciplinary Mathematics. Springer, Singapore, pp. 15-31, 2019. Article (CrossRef Link).

[14] C. Long and S. Datta, "Wavelet based feature extraction for phoneme recognition," in Proc. of Fourth International Conference on Spoken Language Processing. ICSLP '96, 1996. Article (CrossRef Link).

[15] X. Li, X. Yao, J. Jefferys and J. Fox, "Computational Neuronal Oscillations using Morlet Wavelet Transform," in Proc. of 2005 IEEE Engineering in Medicine and Biology 27th Annual Conference, 2006. Article (CrossRef Link). 


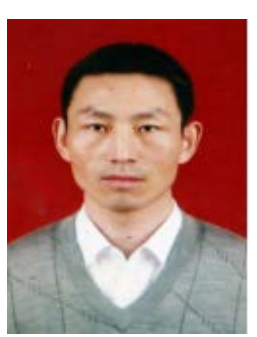

Wenzheng Yu is a professor in the School of Applied Meteorology at Nanjing University of Information Science \& Technology and a member of the Wind Energy and Solar Energy Standard Committee of China Meteorological Administration. His research focuses on meteorological disaster prevention and mitigation, and has directed 1 key R\&D project of the Ministry of Science and Technology, 1 project from National Natural Science Foundation of China, 1 major international cooperation project, and 8 provincial and ministerial projects.

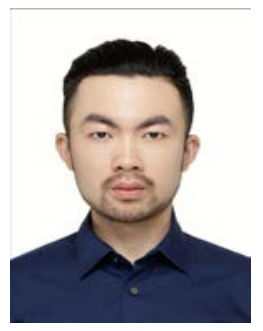

Hanxiaoya Zhang is currently a postgraduate student at the University of Auckland since March 2020. After receiving a Bachelor of Science in Food Science from McGill University, he worked as a research assistant at Jiangsu Academy of Agricultural Sciences and demonstrated great understanding and interest in statistical analyses and their applications in scientific subjects.

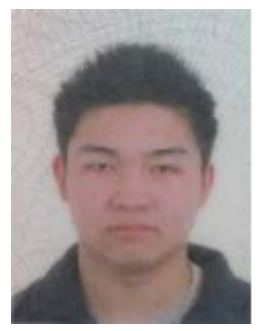

Tianliang Chen received a master's degree in Environmental Engineering at Nanjing University of Information Science \& Technology. His research mostly focuses on the effect of urbanization on climate change.

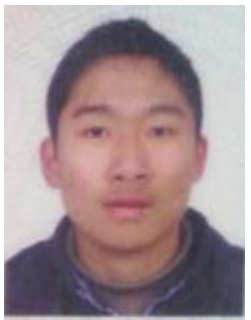

Jing Liu is currently a postgraduate student in the School of Applied Meteorology at Nanjing University of Information Science \& Technology. His research interest involves in climate change and desertification.

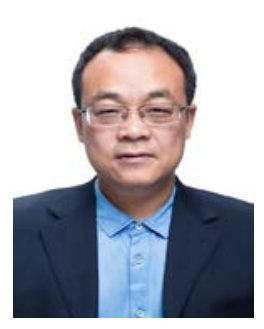

Yanbo Shen is a professor at the Public Meteorological Service Center of China Meteorological Administration, as well as a Chief Scientist of China Meteorological Administration. His research focuses on climatic resources and climate change. 